\title{
Percentual de degradação das forças liberadas por ligaduras elásticas
}

\author{
Emanoela Volles de Souza*, Alvaro de Moraes Mendes**, Marco Antônio de Oliveira Almeida***, \\ Cátia Cardoso Abdo Quintão****
}

\begin{abstract}
Resumo
Objetivo: avaliar ligaduras elásticas da marca Morelli, dos tipos modular e bengala, nas cores cinza e cristal para determinação do percentual de degradação das forças geradas pelas mesmas. Metodologia: as ligaduras elásticas foram estiradas em estiletes de aço inoxidável com diâmetro de $4 \mathrm{~mm}$, imersas em solução de saliva artificial a $37^{\circ} \mathrm{C}$, sendo a intensidade das forças liberadas medidas nos tempos de zero hora, 24 horas, 1 semana, 2 semanas, 3 semanas e 4 semanas, em máquina de ensaios de tração. Resultados e Conclusões: as ligaduras do tipo bengala liberaram, em média e no período de 4 semanas, maior intensidade de força que as do tipo modular. O percentual de degradação das forças liberadas por todas as ligaduras aumentou de forma acentuada entre zero e 24 horas e de forma gradativa após este período, com exceção do $28^{\circ}$ dia quando, provavelmente, houve perda das propriedades elásticas das ligaduras. Não foi observado um comportamento constante das ligaduras bengala e modular em relação às cores cinza e cristal.
\end{abstract}

Palavras-chave: Ligadura elástica (modular). Ligadura elástica (bengala). Degradação da força.

\section{INTRODUÇÃO}

Para amarrar os arcos ortodônticos aos braquetes os ortodontistas dispõem de fios de amarrilho metálico, elastômeros sintéticos circulares ou de braquetes autoligados ${ }^{18}$. No entanto, o uso de ligaduras elásticas tem prevalecido entre grande parte dos ortodontistas, por serem práticas e eficientes. Além de serem colocadas facilmente e rapidamente, estão disponíveis em uma grande variedade de cores e são confortáveis para o paciente $^{18}$. Esse conforto se deve principalmente às suas superfícies lisas, que não produzem irritação tecidual, ao contrário dos amarrilhos metálicos, os quais podem lesar a mucosa bucal quando mal adaptados ${ }^{21}$.

Os elastômeros possuem propriedades excelentes, dentre as quais pode-se destacar a capacidade de se distender e retrair rapidamente, a alta resistência e o alto módulo de elasticidade, quando distendidos, e a recuperação total da tensão sofrida ${ }^{4}$. Porém, os elastômeros não são elásticos ideais e são afetados pela duração da força a eles aplicada e pelo meio ambiente a que são subme$\operatorname{tidos}^{19}$. Já em relação ao percentual de degradação da força gerada quando esses materiais são distendidos e mantidos em torno dos braquetes

* Especialista em Ortodontia e Ortopedia Facial pela UERJ.

** Mestre e Doutor em Ortodontia pela FO-UFRJ. Professor Adjunto da Disciplina de Ortodontia FO-UERJ.

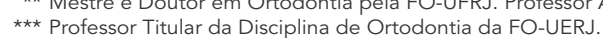

${ }_{\star \star \star \star \star}$ Mestre e Doutora em Ortodontia pela FO-UFRJ. Professora Adjunta da Disciplina de Ortodontia da FO-UERJ. 
por um certo tempo, estudos mostram que esse percentual é alto principalmente nas primeiras 24 horas, diminuindo mais lentamente após esse período ${ }^{1,18,20,21}$. Portanto, o conhecimento sobre as alterações nas propriedades mecânicas das ligaduras elásticas quando estiradas é de grande interesse para o emprego desses materiais, uma vez que poderão permanecer por um tempo relativamente longo na boca dos pacientes, sendo extremamente desejável que, durante esse intervalo, continuem exercendo uma força adequada clinicamente.

$\mathrm{O}$ presente estudo avaliou a intensidade e o percentual de degradação das forças liberadas por ligaduras elásticas dos tipos bengala e modular nas cores cinza e cristal, quando mantidas distendidas e imersas em solução de saliva artificial por 24 horas, 7, 14, 21 e 28 dias.

\section{MATERIAL E MÉTODOS}

Neste estudo foram utilizadas ligaduras elásticas do tipo bengala nas cores cristal (Morelli, cód. 60.03.300) e cinza (Morelli, cód. 60.03.301) e ligaduras elásticas do tipo modular nas cores cristal (Morelli, cód. 60.06.100) e cinza (Morelli, cód. 60.06.101). As ligaduras elásticas foram divididas em 4 grupos: grupo 1 - 60 ligaduras elásticas do tipo modular na cor cinza; grupo 2 - 60 ligaduras elásticas do tipo modular na cor cristal; grupo 3 60 ligaduras elásticas do tipo bengala na cor cinza; e grupo $4-60$ ligaduras elásticas do tipo bengala na cor cristal.

Para a realização dos procedimentos experimentais, as ligaduras elásticas foram devidamente colocadas em estiletes de aço inoxidável (Cavalo - Aços Especiais) com comprimento de $10 \mathrm{~cm}$, afilados ao final de suas extremidades e com diâmetro de $4 \mathrm{~mm}$, equivalente ao diâmetro de um fio de amarrilho utilizado na fixação de um arco com 0,020" de diâmetro em um braquete de incisivo central superior (Morelli, código 10.30.101), como visto na figura 1 . Em seguida, os conjuntos estiletes/ligaduras foram imersos em solução de saliva artificial com $\mathrm{pH}=7,0$ (Farmácia Univer- sitária - Faculdade de Farmácia da Universidade Federal do Rio de Janeiro -UFRJ) e mantidos em uma estufa (estufa bacteriológica Fanem), regulada em torno de $37^{\circ} \mathrm{C}$, pelos períodos de 24 horas, 7 dias, 14 dias, 21 dias e 28 dias.

Para a avaliação da intensidade da força gerada, dez elásticos de cada grupo (controle) tiveram suas forças iniciais medidas logo após a retirada de suas embalagens originais em uma máquina de ensaios de tração, modelo DL-500 MF (EMIC - Equipamentos e Sistemas de Ensaios LTDA), do laboratório de ensaios físicos e mecânicos da Faculdade de Odontologia da Universidade do Estado do Rio de Janeiro - UERJ (Fig. 2). Essa máquina é equipada com transdutores de medição de forças ou strain-gauges (EMIC - Equipamentos e Sistemas de Ensaios LTDA), correspondente a uma carga de 1 Newton (equivalente a 9.80665Kgf) e pinos com $0,9 \mathrm{~mm}$ de diâmetro para fixação das ligaduras elásticas (Fig. 2). Todas as ligaduras elásticas foram distendidas de uma distância inicial de $1 \mathrm{~mm}$ para uma distância final de $5 \mathrm{~mm}$ através da máquina de ensaios de tração, sendo que a velocidade de distensão adotada foi de $5,08 \mathrm{~mm}$ por minuto $(0,2$ polegadas por minuto), conforme descrito por Kovatch $^{12}$.

Após a imersão em solução de saliva artificial e permanência em estufa a $37^{\circ} \mathrm{C}$, pelos intervalos de tempo de 24 horas, 7, 14, 21 e 28 dias, as ligaduras elásticas foram retiradas dos estiletes e imediatamente posicionadas nos pinos da máquina de ensaios de tração para serem avaliadas em relação à intensidade das forças liberadas. Para cada grupo foi aplicado o teste $t$ de Student, para comparação das variáveis: tempo de imersão, tipo e cor dos

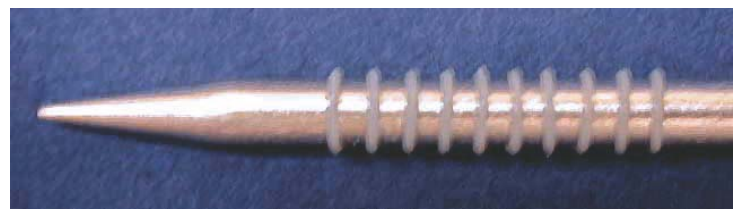

FIGURA 1 - Ligaduras adaptadas ao estilete de aço inoxidável. 

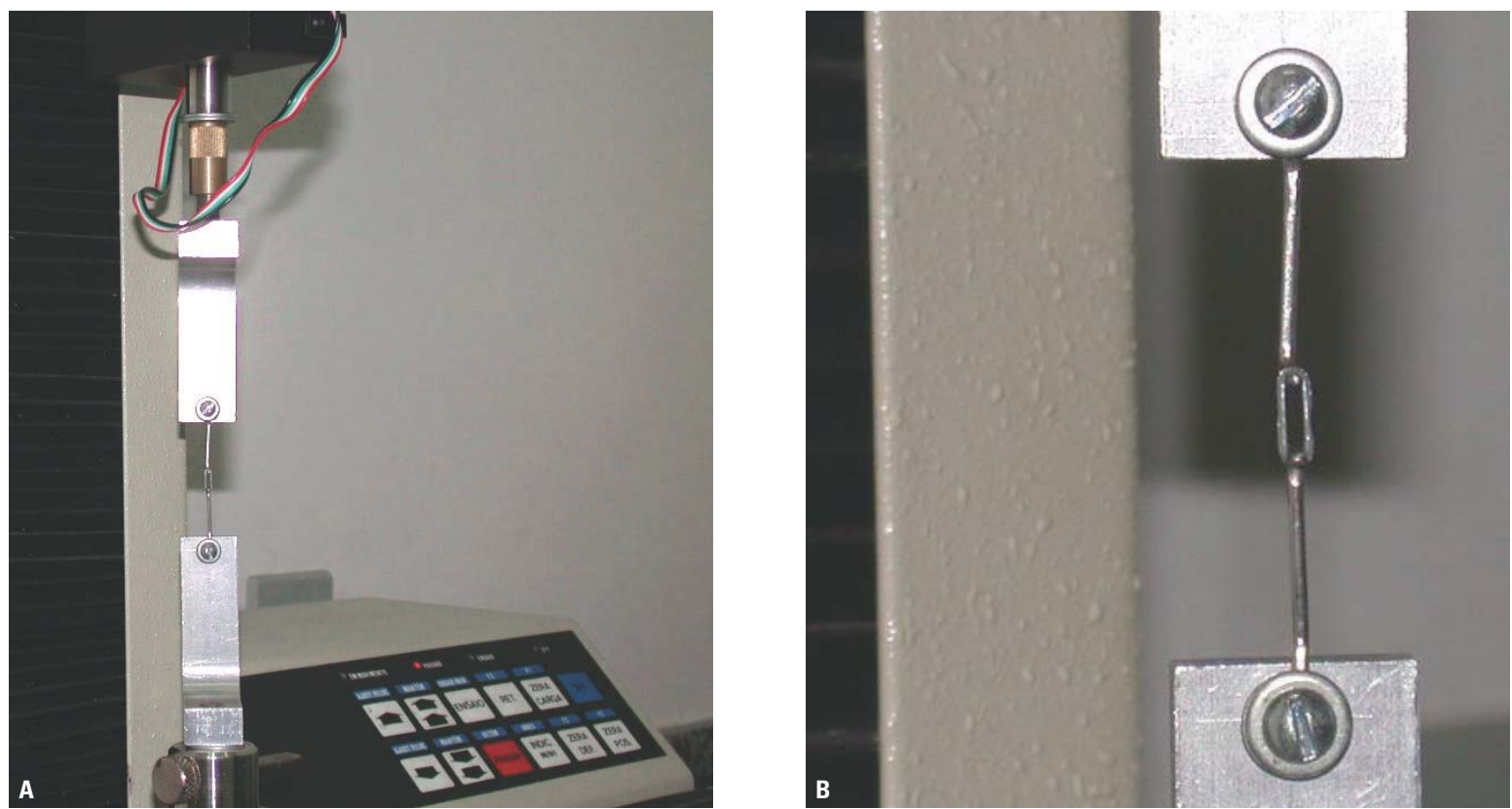

FIGURA 2 - A) Máquina de ensaios de tração com garras adaptadas. B) ligadura elástica sendo distendida.

elásticos; sendo que os níveis adotados de significância foram de $1 \%$ e $5 \%$.

\section{RESULTADOS E DISCUSSÃO}

Os resultados demonstram uma queda progressiva nos valores médios das forças liberadas por ligaduras elásticas dos tipos modular e bengala, nas cores cinza e cristal, durante o período de experimento. Conforme pode ser visto na tabela 1 e no gráfico 1 , a queda mais acentuada nos valores médios das forças ocorreu entre os tempos de zero hora e 24 horas, seguida por uma queda gradativa até a terceira semana de experimento e discreto aumento na quarta semana. Estes achados corroboram com os de Taloumis et al. ${ }^{18}$ ao analisarem ligaduras elásticas e com os de vários outros autores $^{1,2,5,8,9,11,13,15,16,21}$, ao analisarem elásticos em cadeia.

Uma das causas da degradação destes materiais é o fenômeno denominado relaxação, que é a tendência de decréscimo da força liberada em função do tempo ${ }^{2}$. A queda na intensidade das forças liberadas no período entre zero e 24 horas, correspondeu, para as ligaduras do tipo modular, a uma degradação média de $73,86 \%$ (cinza) e de $58,52 \%$ (cristal) e, para as ligaduras do tipo bengala, a uma degradação média de $34,70 \%$ (cinza) e de $46,67 \%$ (cristal). Já no período entre zero hora e 3 semanas a degradação média para as ligaduras do tipo modular foi de $78,59 \%$ (cinza) e de $84,09 \%$ (cristal) e a degradação média para as ligaduras do tipo bengala foi de $55,83 \%$ (cinza) e de $55,68 \%$ (cristal). Esses dados estão de acordo com Andreasen e Bishara ${ }^{1}$, os quais observaram que a força liberada por elásticos em cadeia decresceu mais acentuadamente no primeiro dia de experimento, chegando a uma queda de $55,7 \%$ da força liberada inicialmente na primeira hora e a $74,2 \%$ após 24 horas. Wong ${ }^{21}$ testou elásticos em cadeia produzidos pela Ormco e pela Unitek e obteve resultados semelhantes, observando uma degradação significativa no primeiro dia $(50 \%$ e $73 \%$ respectivamente) e uma queda nos valores das forças liberadas de maneira mais lenta até o vigésimo primeiro dia do experimento. Josell, Leiss e Rekow ${ }^{11}$ avaliando elásticos em cadeia das marcas Ameri- 
Tabela 1 - Forças médias geradas por ligaduras elásticas do tipo modular e bengala das cores cinza e cristal, distribuídas pelos diferentes períodos de tempo de imersão em solução de saliva artificial.

\begin{tabular}{|c|c|c|c|c|c|c|c|}
\hline & & \multicolumn{6}{|c|}{ forças médias geradas (gf) } \\
\hline \multicolumn{2}{|c|}{ amostra } & \multirow{2}{*}{ tempo zero hora } & \multicolumn{5}{|c|}{ tempo de imersão } \\
\hline & & & 24 horas & 1 semana & 2 semanas & 3 semanas & 4 semanas \\
\hline \multirow{2}{*}{ modular } & cinza & 528 & 138 & 156 & 145 & 113 & 165 \\
\hline & cristal & 528 & 219 & 121 & 137 & 84 & 267 \\
\hline \multirow{2}{*}{ bengala } & cinza & 634 & 414 & 274 & 288 & 280 & 357 \\
\hline & cristal & 677 & 361 & 303 & 279 & 300 & 278 \\
\hline
\end{tabular}

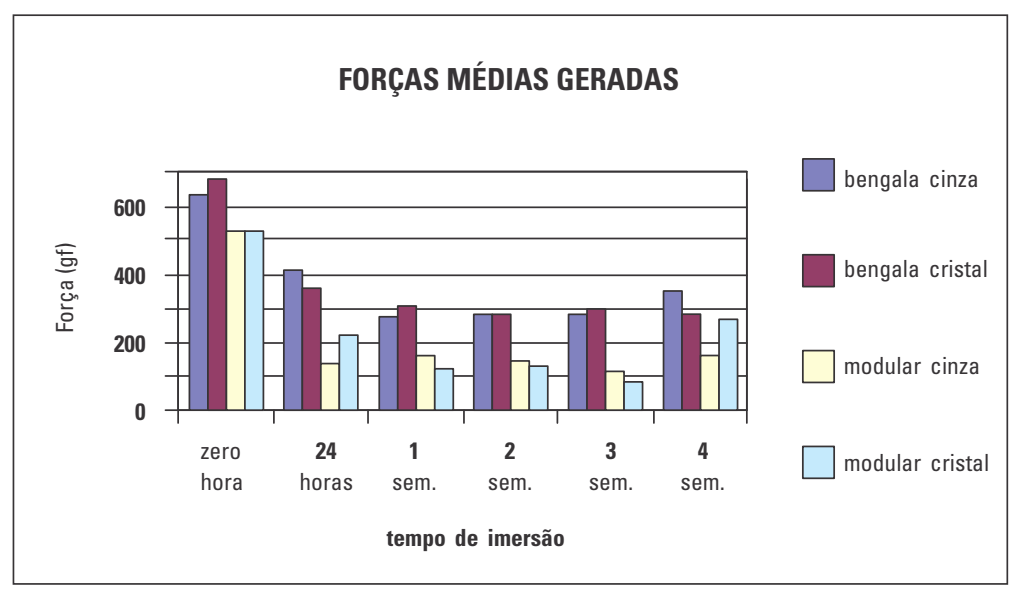

GRÁFICO 1 - Representação gráfica das forças médias geradas por ligaduras elásticas dos tipos bengala e modular das cores cinza e cristal, em relação aos diferentes períodos de tempo de imersão em solução de saliva artificial.

Tabela 2 - Análise das ligaduras dos tipos modular e bengala, nas cores cinza e cristal, em relação às comparações entre os diferentes períodos de tempo de imersão em solução de saliva artificial.

\begin{tabular}{|c|c|c|c|c|c|c|c|}
\hline \multicolumn{2}{|c|}{ ligadura } & $\begin{array}{l}\text { valores (gf) } \\
\text { teste } T\end{array}$ & \multicolumn{5}{|c|}{ comparações (tempos) } \\
\hline \multirow{2}{*}{ modular } & cinza & teste $\mathrm{t}$ & $p=34,311^{* *}$ & $\mathrm{p}=4,500^{* *}$ & $p=0,630$ & $p=1,804$ & $p=2,292^{*}$ \\
\hline & cristal & valores & $528 \times 219$ & $219 \times 121$ & $121 \times 137$ & $137 \times 84$ & $84 \times 267$ \\
\hline \multirow{4}{*}{ bengala } & \multirow{2}{*}{ cinza } & valores & $634 \times 414$ & $414 \times 274$ & $274 \times 288$ & $288 \times 280$ & $280 \times 357$ \\
\hline & & teste t & $p=20,533^{* *}$ & $\mathrm{p}=20,917^{* *}$ & $p=0,911$ & $p=0,384$ & $\mathrm{p}=3,629^{* *}$ \\
\hline & \multirow{2}{*}{ cristal } & valores & $677 \times 361$ & $361 \times 303$ & $303 \times 279$ & $279 \times 300$ & $300 \times 278$ \\
\hline & & teste $\mathrm{t}$ & $\mathrm{p}=32,768^{* *}$ & $p=10,097^{* *}$ & $p=1,755$ & $p=1,504$ & $p=4,348^{* *}$ \\
\hline
\end{tabular}


can Orthodontics, Dentaurum, GAC, Ormco, Rocky Mountain e TP Orthodontics, verificaram que a maior queda na intensidade das forças liberadas ocorreu na primeira hora e que os níveis de liberação das forças após 28 dias variaram de $85 \%$ a 30\% do valor liberado inicialmente, dependendo do grupo testado. Estes resultados também estão de acordo com os de Taloumis et al. ${ }^{18}$, que avaliaram ligaduras elásticas das marcas A Company, American Orthodontics, GAC International, Ormco, Rocky Mountain Orthodontics, TP Orthodontics, e Unitek, constatando uma diminuição nos valores das forças liberadas com o decorrer do tempo, sendo que o percentual de degradação variou de $55 \%$ a $68 \%$ nas primeiras 24 horas de ativação e continuou apresentando uma queda em seus valores até o $28^{\circ}$ dia, só que de forma gradativa. Nesse estudo foi constatado que na quarta semana houve um aumento significativo no valor da força liberada por todas as ligaduras em relação aos valores observados na terceira semana, com exceção da ligadura bengala da cor cristal, que apresentou uma queda em seus valores (Tab. 1, 2). Este comportamento pode ser justificado, provavelmente, pela perda das propriedades elásticas das ligaduras, tornando-as mais rígidas e, conseqüentemente, gerando valores de força maiores, conforme considerado por Wong ${ }^{21}$.

Conforme pode ser visto na tabela 2 , as comparações dos valores médios das forças liberadas nos diferentes períodos de tempo em relação às ligaduras elásticas dos tipos modular e bengala nas cores cinza e cristal, apresentaram resultados significativos entre os períodos zero hora e 24 horas e entre 24 horas e 1 semana, periodos nos quais o percentual de degradação das ligaduras elásticas foi mais acentuado e conseqüentemente apresentou uma queda acentuada nos valores médios das forças liberadas. Já os resultados correspondentes às comparações entre os períodos 1 semana e 2 semanas e entre 2 semanas e 3 semanas, apresentaram valores não significativos, indicando uma queda discreta nos valores das forças liberadas entre a primeira e a terceira semanas de experimento. Exceção foi observada para a ligadura do tipo modular na cor cristal, que apresentou um resultado significativo na comparação das forças médias entre a segunda e a terceira semanas. Esta variação pode ser justificada pelo fato de que a qualidade desses materiais está na dependência do processo de fabricação empregado, visto que a composição das ligaduras é resultante do grau de tecnologia empregada, refinamento da técnica de obtenção e pela qualidade das matérias primas utilizadas durante a confecção do material ${ }^{10,14,17}$. Desse modo, se não houver um rígido controle de qualidade quando da fabricação dos elásticos, os resultados de testes similares podem ser diferentes, uma vez que, em um mesmo lote podem ser encontradas diferenças nas propriedades das ligaduras elásticas, conforme constatado e relatado por Wong ${ }^{21}$. Já o aumento observado na quarta semana também pode ser atribuído, provavelmente, às alterações nas propriedades físicas das ligaduras, conforme considerado anteriormente.

Alterações nas propriedades dos elastômeros também podem ser decorrentes do aumento de volume e da descoloração quando expostos ao meio bucal, os quais são ocasionados pelo preenchimento dos espaços na matriz da borracha por fluidos e bactérias ${ }^{6,7,16,21}$. Dessa forma, nesse estudo, o aumento de volume em decorrência da absorção de líquidos pode ter favorecido a queda nos valores médios das forças liberadas pelas ligaduras elásticas das cores cinza e cristal e dos tipos modular e bengala, conforme pode ser visto pelos resultados contidos na tabela 1 e de acordo com os achados de Ferretier, Meyer e Lorton ${ }^{6}$ e Wong ${ }^{21}$.

Quando as ligaduras elásticas das cores cristal e cinza foram analisadas em relação às comparações das forças médias liberadas pelos tipos bengala e modular, foi constatado (Tab. 3), que os resultados foram estatisticamente significativos para todas as comparações realizadas nos períodos de zero hora, 24 horas, 1 semana, 2 semanas, 3 semanas e 4 semanas. Portanto, as ligaduras do tipo benga- 
la apresentaram uma intensidade de força maior que as do tipo modular, durante todo o período do experimento (zero hora até 4 semanas). Este comportamento foi inversamente proporcional ao percentual de degradação, ou seja, as ligaduras do tipo bengala apresentaram menor percentual de degradação que as do tipo modular nos tempos considerados. Este fato pode ser justificado pela diferença na configuração da secção transversal das ligaduras, de modo que a modular tem secção circular e a bengala secção retangular e, conseqüentemente, maior quantidade de material (Fig. 3). Além disso, a forma de manufatura do material pode ter influenciado nos resultados, já que a ligadura modular é feita pelo método de injeção em moldes e a bengala pelo método de extrusão, conforme relatado por Morton ${ }^{17}$.

Para minimizar a influência de certos tipos de alimentos no meio bucal, que possam causar alterações na coloração dos elastômeros, são incluídas pigmentações metálicas às ligaduras durante o processo de manufatura, o que reduz o nível de força liberada e as suas propriedades elásticas ${ }^{21}$. Dessa forma as diversas cores poderiam alterar diferentemente as propriedades das ligaduras. Lu et al..$^{14}$ observaram que os elásticos em cadeia transparentes apresentaram resultados estatisticamente melhores que os da cor cinza, gerando forças mais favoráveis para o uso clínico. No entanto, na análise

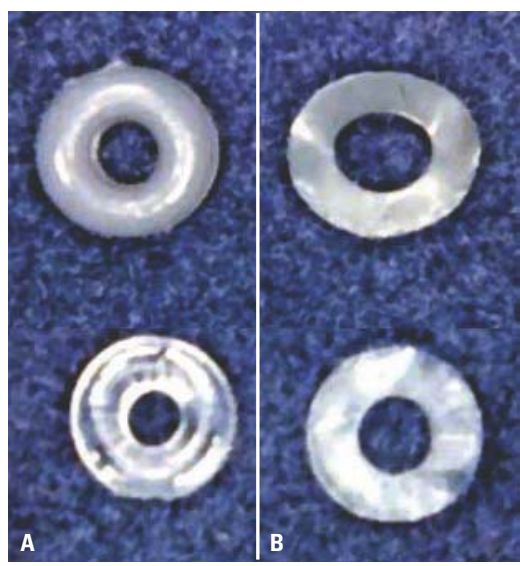

FIGURA 3 - A) ligaduras elásticas do tipo modular. B) ligaduras elásticas do tipo bengala. Nota-se uma nítida diferença na configuração dos dois materiais.

Tabela 3 - Análise das ligaduras elásticas das cores cristal e cinza em relação às comparações entre as forças médias liberadas pelos tipos bengala e modular, nos diferentes períodos de tempo de imersão em solução de saliva artificial.

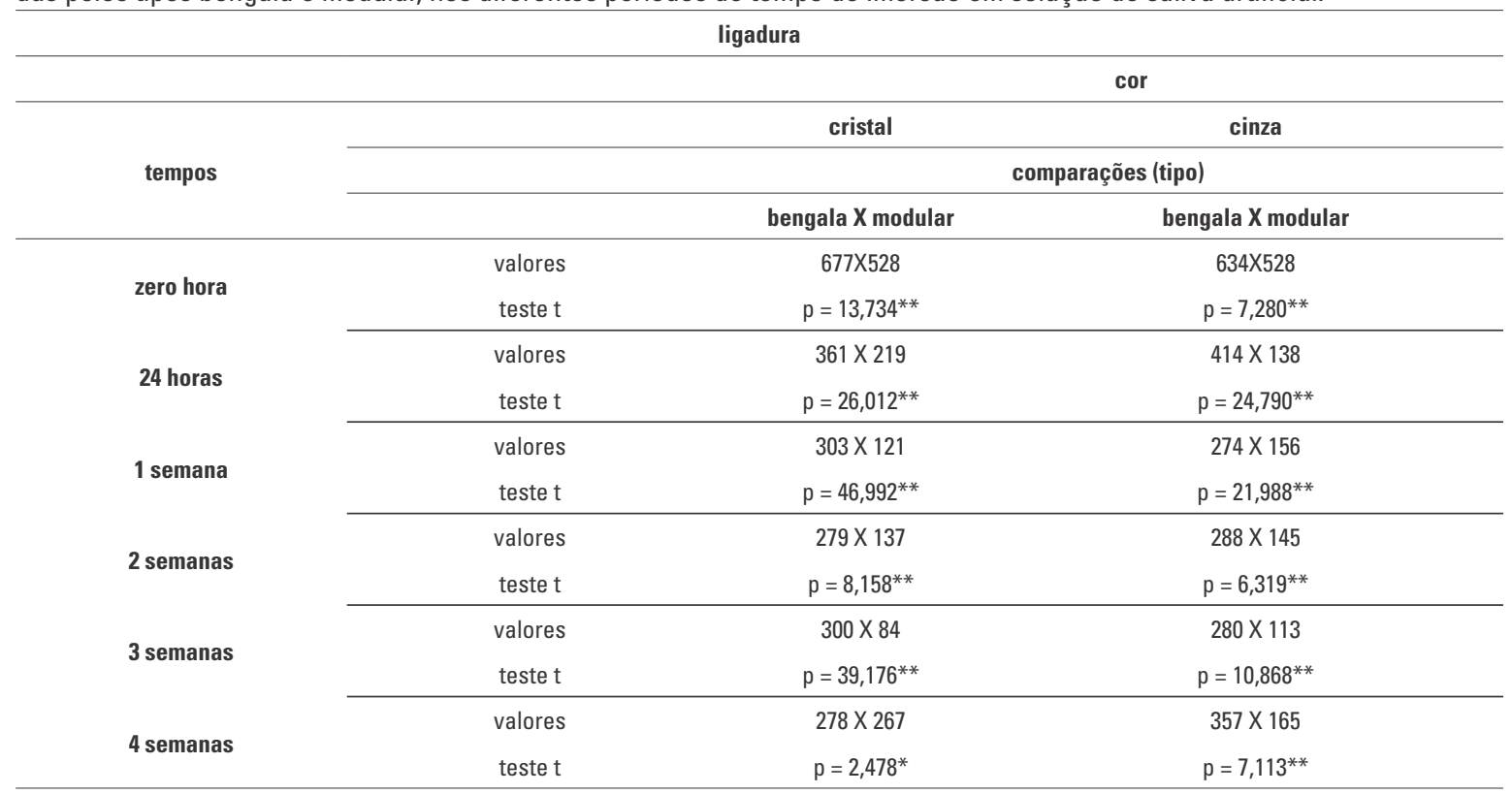

${ }^{*}=p \leq 0,05 e^{* *}=p \leq 0,01$. 
Tabela 4 - Análise das ligaduras elásticas dos tipos bengala e modular em relação às comparações entre as forças médias liberadas pelas cores cinza e cristal, nos diferentes períodos de tempo de imersão em solução de saliva artificial.

\begin{tabular}{|c|c|c|c|}
\hline \multirow{5}{*}{ tempos } & & \multicolumn{2}{|l|}{ ligadura } \\
\hline & & \multicolumn{2}{|c|}{ tipo } \\
\hline & & bengala & modular \\
\hline & & \multicolumn{2}{|c|}{ comparações (cor) } \\
\hline & & cinza $X$ cristal & cinza $X$ cristal \\
\hline \multirow{2}{*}{ zero hora } & valores & $634 \times 677$ & $528 \times 528$ \\
\hline & teste t & $p=3,373^{* *}$ & $p=0,000$ \\
\hline \multirow{2}{*}{24 horas } & valores & $414 \times 361$ & $138 \times 219$ \\
\hline & teste $\mathrm{t}$ & $\mathrm{p}=7,875^{* *}$ & $p=19,941^{* *}$ \\
\hline \multirow{2}{*}{1 semana } & valores & $274 \times 303$ & $156 \times 121$ \\
\hline & teste $\mathrm{t}$ & $\mathrm{p}=5,087^{* *}$ & $\mathrm{p}=10,412^{* *}$ \\
\hline \multirow{2}{*}{2 semanas } & valores & $288 \times 279$ & $145 \times 137$ \\
\hline & teste $\mathrm{t}$ & $p=0,455$ & $p=0,388$ \\
\hline \multirow{2}{*}{3 semanas } & valores & $280 \times 300$ & $113 \times 84$ \\
\hline & teste $\mathrm{t}$ & $p=1,296$ & $p=5,432^{* *}$ \\
\hline \multirow{2}{*}{4 semanas } & valores & $357 \times 278$ & 165 x 267 \\
\hline & teste $\mathrm{t}$ & $p=5,124^{* *}$ & $\mathrm{p}=4,514^{* *}$ \\
\hline
\end{tabular}

${ }^{*}=p \leq 0,05 e^{* *}=p \leq 0,01$.

das ligaduras do tipo bengala e modular, em relação às comparações entre as forças médias liberadas pelas cores cinza e cristal, foi constatado que os resultados foram significativos para os tipos bengala e modular nos períodos de 24 horas, 1 semana e 4 semanas, não-significativos na segunda semana e significativo, somente para o tipo bengala em zero hora e para o tipo modular na terceira semana (Tab. 4). Assim sendo, essa variação caracteriza um comportamento heterogêneo em relação às comparações realizadas entre as cores cinza e cristal, indicando que o fator cor pode não ter influenciado nos resultados de forma constante e homogênea. Estes achados estão de acordo com os encontrados por Baty, Volz e Fraunhofer ${ }^{3}$, que demonstraram que os elásticos em cadeia coloridos apresentaram valores de força equivalentes aos elásticos cinza e, em média, todos obtiveram valores referentes ao percentual de degradação da força liberada que não comprometeriam o seu desempenho clínico.

\section{CONCLUSÕES}

As ligaduras do tipo bengala (cinza e cristal) liberaram, em média e no período de 4 semanas, maior intensidade de forças que as do tipo modular.

O percentual de degradação das forças liberadas por todas as ligaduras aumentou de forma acentuada entre zero e 24 horas e de forma gradativa nos períodos correspondentes a 7, 14 e 21 dias, com exceção do $28^{\circ}$ dia, quando, provavelmente, houve perda das propriedades elásticas das ligaduras.

A intensidade das forças liberadas por todas as ligaduras diminuiu de forma acentuada entre zero e 24 horas, de forma gradativa de 24 horas até o $21^{\circ}$ dia e apresentou um discreto aumento no $28^{\circ}$ dia de experimento.

Não foi observado um comportamento constante para as ligaduras bengala e modular em relação às cores cinza e cristal.

Enviado em: agosto de 2006 Revisado e aceito: setembro de 2006 


\title{
Force decay rate of orthodontic elastomeric ligatures
}

\begin{abstract}
Purpose: to evaluate gray and clear module and cane-loaded elastomeric ligatures of the brand Morelli to determine the percentage of decay of the forces they deliver. Methods: the elastomeric ligatures were stretched over $4 \mathrm{~mm}$ diameter stainless steel dowels and placed in synthetic saliva bath at $37^{\circ} \mathrm{C}$. The intensity of the forces delivered were taken at start, after 24 hours, one week, two weeks, three weeks and four weeks at a tensile test machine. Results and Conclusions: the cane-loaded ligatures delivered, in a period of 4 weeks, in average, greater force intensity than the modules. The percentage of force decay of all ligatures increased rapidly between zero and 24 hours and gradually after that period, except for the 28th day, when probably there was a loss of elastic properties of the ligatures. The researchers did not note any constant behavior in the cane-loaded and module ligatures in relation to the colors: gray and clear.
\end{abstract}

Key words: Elastomeric ligature (module). Elastomeric ligature (cane-loaded). Force decay.

\section{REFERÊNCIAS}

1. ANDREASEN, G. F.; BISHARA, S. E. Comparison of alastik chains with elastics involved with intra-arch molar to molar forces. Angle Orthod., Appleton, v. 40, no. 3, p. 151-158, July 1970.

2. ASH, J. L.; NIKOLAI, R. J. Relaxation of orthodontic elastomeric chains and modules in vitro and in vivo. J. Dent. Res. Baltimore, v. 56, no. 5/6, p. 685-690, May/July 1978

3. BATY, D. L.; VOLZ, J. E.; FRAUNHOFER, J. A. Force delivery properties of colored elastomeric modules. Am. J. Orthod. Dentofacial Orthop., St. Louis, v. 6, no. 1, p. 40-46, July 1994

4. BILLMEYER JR., F. N. Textbook of polymer science. 3rd ed. New York: J. Wiley, 1984.

5. BRANTLEY, W. A.; SALANDER, S.; MYERS, C. L.; WINDERS, R. $V$. Effect of prestretching on force degradation characteristics of plastic modules. Angle Orthod., Appleton, v. 49, no. 1, p. 37-43, Jan.1979.

6. FERRITER, J. P.; MEYER, C. E.; LORTON, L. The effect of hydrogen ion concentration on the force-degradation rate of orthodontic polyurethane chain elastics. Am. J. Orthod. Dentofacial Orthop., St. Louis, v. 8, no. 5, p. 404-410, Nov. 1990.

7. FRAUNHOFER, J. A.; COFFELT, M. T. P.; ORBELL, G. M. The effects of artificial saliva and topical fluoride treatments on the degradation of the elastic properties of orthodontic chains. Angle Orthod., Appleton, v. 62, no. 4, p. 265-274, Oct./Dec.1992.

8. GENOVA, D. C. D.; McINNES-LEDOUX, P.; WEINBERG, R.; SHAYE, R. Force degradation of orthodontic elastomeric chains: a product study. Am. J. Orthod. Dentofacial Orthop., St. Louis, v. 87, no. 5, p. 377-384, May 1985.

9. HERSHEY, H. G.; REYNOLDS, G. W. The plastic module as an orthodontic tooth-moving mechanism. Am. J. Orthod., St. Louis, v. 67, no. 5, p. 554-562, May 1975

10. HUGET, E. F.; PATRICK, K. S. Observations on the elastic behavior of a synthetic orthodontic elastomer. J. Dent. Res., Baltimore, v. 69, no. 2, p. 496-501, Nov. 1990.

11. JOSELL, S. D.; LEISS, J. B.; REKOW, D. Force degradation in elastomeric chain. Semin. Orthod., Birmingham, v. 3, no. 3, p. 189-197, Sept. 1997

12. KOVATCH, J. S.; LAUTENSCHLANGER, E. P.; APFEL, D. A. KELLER, J. C. Load-extension-time behavior of orthodontic alastiks. J. Dent. Res., Baltimore, v. 55, no. 5, p. 783-786, Sept./Oct. 1976.

13. KUSTER, R.; INGERVALL, B.; BÜRGIN, W. Laboratory and intraoral tests of the degradation of elastic chains. Eur. J. Orthod., London, v. 8, p. 202-208, 1986.
14. LU, T. C., WANG, W. N., TARNG, T. H.; CHEN. J. W. Force de cay of elastomeric chain-a serial study. Part II. Am. J. Orthod. Dentofacial Orthop., St. Louis, v. 104, no. 4, p. 373-377, Oct.1993.

15. MATTA, E. N. R. Avaliação laboratorial da deformação plástica e da capacidade de liberação de força por elásticos plásticos. 1996. 86 f. Dissertação (Mestrado em Ortodontia)-Universidade Federal do Rio de Janeiro, Rio de Janeiro, 1996.

16. MATTA, E. N. R.; CHEVITARESE, O. Avaliação laboratorial da força liberada por elásticos plásticos. Rev. SBO, Rio de Janeiro, v. 3, n. 4, p. 131-136, ago.1997

17. MORTON, M. Rubber technology. 3rd. ed. Londres: Chapman \& Hall, 1995.

18. TALOUMIS, L. J. et al. Force decay and deformation of orthodontic elastomeric ligatures. Am. J. Orthod. Dentofacial Orthop., St. Louis, v. 111, no. 1, p. 1-11, Jan. 1997.

19. YOUNG, J.; SANDRIK, J. L. The influence of preloading on stress relaxation of orthodontic elastic polymers. Angle Orthod., Appleton, v. 49, no. 2, p. 104-109, Apr. 1979.

20. WARE, A. L. Some properties of plastics modules used for tooth movement. Aust. Orthod. J., Brisbane, v. 2, no. 5, p. 200-201, Feb. 1971

21. WONG, A. K. Orthodontic elastic materials. Angle Orthod. Appleton, v. 46, no. 2, p. 196-205, Apr. 1976.
Endereço para correspondência

Emanoela Volles de Souza

Rua Carlos Gruensch 205, América

CEP: 89.201-745 - Joinville/SC

E-mail: emanoelavolles@terra.com.br 